\section{Evolution of high mutation} rates in experimental populations of E. colf

\author{
Paul D. Sniegowski, Philip J. Gerrish $\dagger$ \\ \& Richard E. Lenski $\dagger$ \\ * Department of Biology, University of Pennsylvania, Philadelphia, \\ Pennsylvania 19104, USA \\ $\dagger$ Center for Microbial Ecology, Michigan State University, East Lansing, \\ Michigan 48824, USA
}

Most mutations are likely to be deleterious, and so the spontaneous mutation rate is generally held at a very low value ${ }^{1}$. Nonetheless, evolutionary theory predicts that high mutation rates can evolve under certain circumstances ${ }^{2-4}$. Empirical observations have previously been limited to short-term studies of the fates of mutator strains deliberately introduced into laboratory populations of Escherichia coli ${ }^{5-7}$, and to the effects of intense selective events on mutator frequencies in $E$. coli ${ }^{8}$. Here we report the rise of spontaneously originated mutators in populations of $E$. coli undergoing long-term adaptation to a new environment. Our results corroborate computer simulations of mutator evolution in adapting clonal populations ${ }^{4}$, and may help to explain observations that associate high mutation rates with emerging pathogen ${ }^{9}$ and with certain cancers ${ }^{10}$.

As a means of studying evolution directly in the laboratory, 12 replicate experimental populations of a clonal strain of $E$. coli were established ${ }^{11}$. Because these populations were founded from a single ancestral clone, mutation provided their only source of genetic variation. The glucose-limited environment in which these populations were propagated was essentially novel at the outset of the experiment and it provided considerable scope for adaptive evolution. Substantial evolutionary increases in fitness and changes in certain other phenotypic features in these populations have been documented elsewhere ${ }^{11-15}$.

We measured mutation rates in the common ancestral strain and in the 12 experimental populations after they had been evolving for 10,000 generations. Most of the populations retained the ancestral mutation rate, but three populations (designated $\mathrm{Ara}^{-} 2 \mathrm{Ara}^{-} 4$ and $\mathrm{Ara}^{+} 3$ ) displayed mutation rates that were between one and two orders of magnitude higher than those in the ancestor (Fig. 1). The times at which mutator phenotypes arose during the evolution of populations $\mathrm{Ara}^{-} 2, \mathrm{Ara}^{-} 4$ and $\mathrm{Ara}^{+} 3$, as determined by screening for the mutator phenotype in isolates stored periodically throughout the experiment, are shown in Fig. 2. Once a lineage displayed a mutator phenotype, all subsequent isolates from that lineage also tested as mutators through 10,000 generations. This observation indicates that mutators rose to and remained at high frequencies in these populations.

To ascertain a genetic basis for the observed mutator phenotypes, we transformed 10,000-generation clonal isolates from populations $\mathrm{Ara}^{-} 2, \mathrm{Ara}^{-} 4$ and $\mathrm{Ara}^{+} 3$, and an isolate from the ancestral strain REL606 with multicopy plasmids bearing wild-type alleles of seven known general mutator loci. The ancestral mutation rate was fully restored in the $\mathrm{Ara}^{-} 2, \mathrm{Ara}^{-} 4$ and $\mathrm{Ara}^{+} 3$ strains only by the presence of wild-type alleles of genes in the methyl-directed mismatch repair pathway ${ }^{16}$ (Fig. 3). The mutation rate in the ancestral strain, REL606, was not significantly affected by the plasmids (with a single exception: see Fig. 3). In the $\mathrm{Ara}^{+} 3$ strain the $m u t S^{+}$allele alone restored the ancestral mutation rate. In the $\mathrm{Ara}^{-} 2$ and $\mathrm{Ara}^{-} 4$ strains the ancestral rate was restored completely by $u v r D^{+}$, and the data also indicated a partial effect of $m u t L^{+}$. Recent studies have suggested that there is a mechanistic interaction between the mutL and $u v r D$ gene products, such that a defect in one may in some cases be complemented by increased production of the other (P. Modrich, personal communication).

Most mutations are deleterious, so mutator alleles are likely on average to have negative effects on fitness. In an evolving clonal population, however, a deleterious mutator can rise to high frequency (hitch-hike) in association with an adaptive mutation, provided that the selective cost of the mutator does not outweigh the selective benefit of the adaptive mutation. Hitch-hiking of mutators with adaptive mutations was demonstrated previously in chemostat populations of E. coli ${ }^{6}$, but these results left open the question of whether such events would be likely to occur in natural populations. When a mutator was introduced above a relatively high threshold number relative to the wild type, the mutator population always acquired an adaptive mutation before the wildtype population, and the mutator rose to high frequency. However, when the mutator was introduced in lower numbers, the wild-type population always acquired a beneficial mutation first and displaced the mutator.

In the evolution experiment we have described here, as in natural populations, mutator alleles must have arisen by mutation. This raises the question of how mutators reached high frequencies in 3 of our 12 populations. Computer simulations ${ }^{4}$ and analytical modelling (P.J.G. and P.D.S., unpublished data) suggest that rare mutators may occasionally hitch-hike to high frequencies in finite asexual populations as a consequence of chance associations with adaptive mutations. We think that such chance hitch-hiking events are the likely explanation for our results. Sufficient time may not have elapsed for them to have been observed in earlier studies of
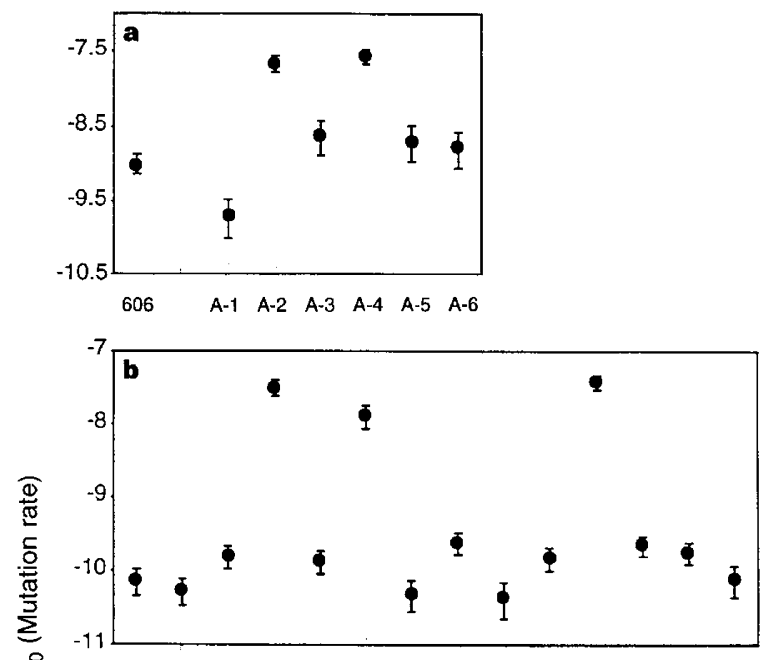

$606 \quad 607$ A-1 A-2 A-3 A-4 A-5 A-6 A+1 A+2 A+3 A+4 A+5 A+6

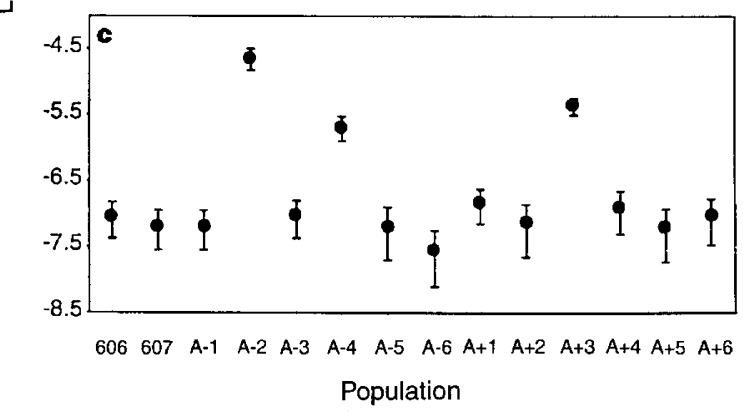

Figure 1 Rates of mutation in $12 \mathrm{Ara}^{-}$and $\mathrm{Ara}^{+}$experimental populations ( $\mathrm{A}-1$ to $A-6$ and $A+1$ to $A+6)$ at 10,000 generations and in their common ancestors REL606 $\left(\mathrm{Ara}^{-}\right)$and REL607 $\left(\mathrm{Ara}^{+}\right)$. Error bars give approximate 95\% confidence intervals. a, Reversion to Ara ${ }^{+}$in isolates from the $7 \mathrm{Ara}^{-}$populations. b, Mutation to nalidixic-acid resistance. c, Mutation to bacteriophage T5 resistance. 
competition between mutator and wild-type strains $s^{5-7}$, as those studies were carried out for only a few tens or hundreds of generations, as opposed to the 10,000 generations in our study. Although we cannot formally exclude the possibility that certain mutations in mismatch repair might increase fitness directly, it is much more likely that the evolved mutators we observed are deleterious or at best effectively neutral. Indeed, known mutator

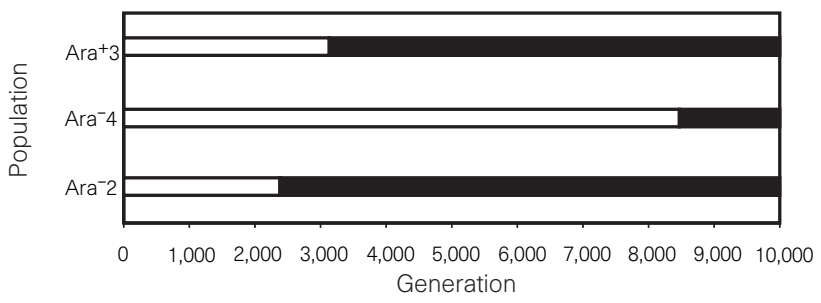

Figure 2 Time of appearance and evolutionary persistence of mutator phenotypes in experimental populations $\mathrm{Ara}^{-} 2, \mathrm{Ara}^{-} 4$ and $\mathrm{Ara}^{+} 3$. White bars, wild type; black bars, mutator.
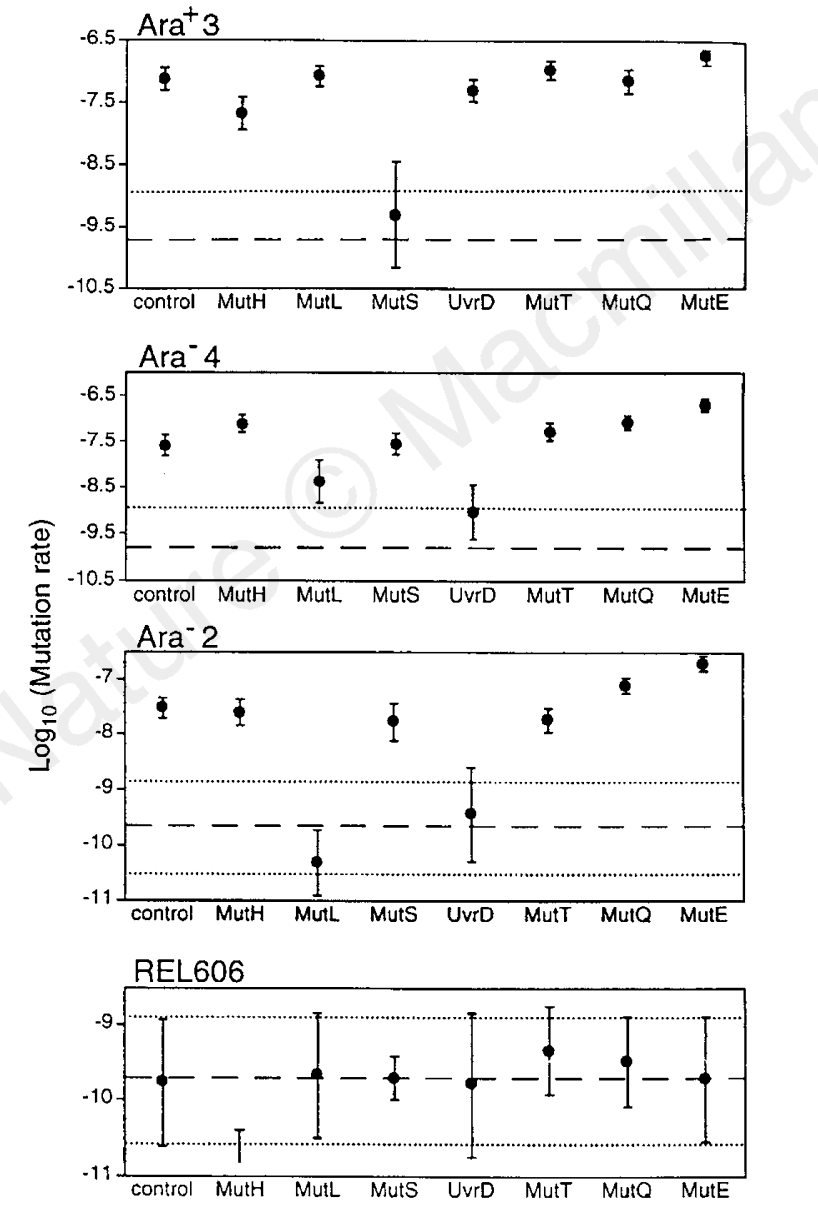

Figure 3 Mutation rates to nalidixic-acid resistance in 10,000-generation isolates from populations $\mathrm{Ara}^{+} 3, \mathrm{Ara}^{-} 4$ and $\mathrm{Ara}^{-} 2$ and ancestor REL606 transformed with plasmids bearing wild-type alleles of seven known general mutator loci. Controls shown are plasmid free. Error bars give approximate 95\% confidence limits. Only an upper confidence limit is shown for the case in which no mutants were obtained in any culture. For visual comparison, the dashed horizontal line and dotted horizontal lines in each panel illustrate the mutation rate and approximate 95\% confidence limits, respectively, measured in the ancestral control. alleles of $m u t S$, mutL and $u v r D$ do not increase cell fitness under our experimental conditions (C. Zeyl, unpublished data).

A prediction of the simulations conducted by Taddei et al. ${ }^{4}$ is that mutators will hitch-hike in some, but not all, finite asexual populations undergoing adaptation. Our experimental observations are consistent with this prediction. A further prediction is that fitness will be higher on average in mutator populations than in those that retain the wild-type mutation rate. However, this fitness effect is very small and subtle: approximately half of the mutator populations in the simulations of Taddei et al. did not show higher fitness, and the observed increases in fitness over contemporaneous wildtype populations were slight (approximately 1\%) in the remainder (see Fig. $3 \mathrm{~b}$ in ref. 4). We tested for a relationship between mutability and fitness in our experimental populations in several ways, none of which gave statistical significance. For example, we tested for a correlation between mutability ranks (as calculated from data obtained in screens for mutator phenotypes; see Methods) and relative fitness ranks averaged across the duration of the experiment in all 12 populations. The results were inconclusive $(r=0.28$, $n=12$, one-tailed $P=0.19)$. It is clear from our experiments and the simulations of Taddei et ll $^{4}$ that increased mutation rate and not increased fitness is the more striking consequence of the hitchhiking process.

Our finding that asexual populations can evolve high mutation rates in a relatively benign environment may help to explain observations associating mismatch repair mutators with certain cancers ${ }^{10}$ and with pathogenicity in E. coli and Salmonella ${ }^{9}$. As in our experimental populations, high mutation rates may evolve as a chance byproduct of adaptation in clonal tumour lineages and in populations of asexual pathogens ${ }^{17,18}$. The potential for faster evolution once high mutation rates have evolved may have important health implications.

\section{Methods}

Experimental system. The common ancestral strain was REL606, an Ara clone of E. coli B obtained by B. R. Levin from S. Lederberg ${ }^{19}$. A spontaneous $\mathrm{Ara}^{+}$revertant was selected from REL606 and designated REL607. Six clones each from REL606 and REL607 were used to found the 12 experimental populations, which were propagated at $37^{\circ} \mathrm{C}$ by daily 100 -fold dilutions into $10 \mathrm{ml}$ of fresh Davis minimal medium ${ }^{20}$ supplemented with glucose at $25 \mu \mathrm{g} \mathrm{ml}^{-1}$. Population sizes fluctuated between approximately $5 \times 10^{6}$ cells after dilution and $5 \times 10^{8}$ cells at the stationary phase. Isolates from each population were stored at $-80^{\circ} \mathrm{C}$ at 100 -generation intervals during the first 2,500 generations of the experiment, and at 500-generation intervals thereafter. Mutation rate measurements. Fluctuation tests ${ }^{21}$ to estimate a given rate of mutation were conducted simultaneously on all strains to provide a controlled comparison. Before testing, strains were revived and regrown for 3 days in the original experimental medium to re-establish the physiological conditions that had prevailed in the evolving populations. In general, 24 or more cultures of a given strain were grown from inocula of approximately 1,000 cells for each fluctuation test. For a given mutation rate measurement, all strains to be compared were grown separately in the same batch of Davis minimal medium. Cultures were grown to stationary phase before selective plating. (An additional $24 \mathrm{~h}$ in stationary phase had no discernible effect on the mutation rate in pilot studies.) Final population sizes were estimated by growing and sampling three extra cultures taken at random for each strain and measuring cell densities using a Coulter particle counter; the means were used in mutation rate calculations. $\mathrm{Ara}^{+}$mutants were enumerated on Davis minimal agar medium supplemented with $4 \mathrm{mg} \mathrm{ml}^{-1}$ arabinose; $\mathrm{Nal}^{\mathrm{r}}$ mutants were enumerated on LB agar medium ${ }^{22}$ containing $20 \mu \mathrm{g} \mathrm{ml}^{-1}$ of nalidixic acid; $\mathrm{T}^{\mathrm{r}}$ mutants were enumerated by plating cultures with excess phage $\mathrm{T} 5 \mathrm{in}$ soft agar. Time of origin and persistence of mutator phenotypes. Mutator screens were conducted at approximately 500-generation intervals over the 10,000 generations of the experiment. Overnight cultures in $5 \mathrm{ml}$ of Davis minimal medium (supplemented with $1,000 \mu \mathrm{g} \mathrm{ml}^{-1}$ glucose) were assayed for $\mathrm{Nal}^{\mathrm{r}}$ mutant numbers at the stationary phase. Expected distributions of mutants, based on mutator and ancestral mutation rates, were used to generate criteria 
by which isolates could be scored as mutator or non-mutator with $>95 \%$ confidence.

Complementation tests. Strains were transformed with plasmids carrying wild-type alleles for $m u t H$ (plasmid pGW1899) ${ }^{23}$, mutL (pGW1842) ${ }^{23}$, mutS $(\mathrm{pgW} 1811)^{23}, u v r D(\mathrm{pGT} 26)^{24}$, mutT $(\mathrm{pSK} 25)^{25}, d_{n a Q}(\mathrm{pMM} 5)^{26}$ and $d n a E$ $(\mathrm{pMK} 9)^{27}$, according to a standard protocol ${ }^{28}$. Fluctuation tests were conducted as described above, except that all strains were propagated in LB medium (containing $60 \mu \mathrm{g} \mathrm{ml}^{-1}$ of ampicillin where the strain was plasmid bearing), and five parallel cultures were used per fluctuation test.

Analysis of fluctuation test data. A local computer program using a LuriaDelbrück distribution-generating algorithm ${ }^{29}$ was used to calculate maximumlikelihood mutation rates from fluctuation test data. Approximate 95\% confidence intervals for the mutation rates illustrated in Fig. 1 were calculated from formulae $\mathrm{e}^{30}$. Approximate $95 \%$ confidence limits for the mutation rates illustrated in Fig. 3 are based on the theoretical variance of the maximumlikelihood estimate of $\ln (m)$, assuming normality, where $m$ is the expected number of mutations per culture.

Received 3 March; accepted 6 May 1997

1. Drake, J. W. Spontaneous mutation. Annu. Rev. Genet. 25, 125-146 (1991).

2. Leigh, E. G. Natural selection and mutability. Am. Nat. 104, 301-305 (1970).

3. Ishii, K., Matsuda, H., Iwasa, Y. \& Sasaki, A. Evolutionarily stable mutation rate in a periodically changing environment. Genetics 121, 163-174 (1989).

4. Taddei, F. et al. Role of mutator alleles in adaptive evolution. Nature 387 700-702 (1997).

5. Cox, E. C. \& Gibson, T. C. Selection for high mutation rates in chemostats. Genetics 77, 169-184 (1974).

6. Chao, L. \& Cox, E. C. Competition between high and low mutating strains of Escherichia coli. Evolution 37, 125-134 (1983).

Tröbner, W. \& Piechocki, R. Competition between isogenic $m u t S$ and $m u t^{+}$populations of Escherichia coli K12 in continuously growing cultures. Mol. Gen. Genet. 198, 175-176 (1984).

8. Mao, E. F., Lane, L., Lee, J. \& Miller, J. H. Proliferation of mutators in a cell population. J. Bacteriol. 179, 417-422 (1997)

9. LeClerc, J. E., Li, B., Payne, W. L. \& Cebula, T. High mutation frequencies among Escherichia coli and Salmonella pathogens. Science 274, 1208-1211 (1996).

10. Modrich, P. Mismatch repair, genetic stability and tumour avoidance. Phil. Trans. R. Soc. Lond. B 347, 89-95 (1995).

11. Lenski, R. E., Rose, M. R., Simpson, S. C. \& Tadler, S. C. Long-term experimental evolution in Escherichia coli. I. Adaptation and divergence during 2,000 generations. Am. Nat. 138, 1315-1341 (1991).

12. Lenski, R. E. \& Travisano, M. Dynamics of adaptation and diversification: A 10,000-generation experiment with bacterial populations. Proc. Natl Acad. Sci. USA 91, 6808-6814 (1994).

13. Vasi, F., Travisano, M. \& Lenski, R. E. Long-term experimental evolution in Escherichia coli. II. Changes in life-history traits during adaptation to a seasonal environment. Am. Nat. 144, 432-456 (1994).

14. Travisano, M. \& Lenski, R. E. Long-term experimental evolution in Escherichia coli. IV. Targets of selection and the specificity of adaptation. Genetics 143, 15-26 (1996).

15. Elena, S. F., Cooper, V. S. \& Lenski, R. E. Punctuated evolution caused by selection of rare beneficial mutations. Science 272, 1802-1804 (1996).

16. Modrich, P. Mechanisms and biological effects of mismatch repair. Annu. Rev. Genet. 25, 229-253 (1991).

17. Nowell, P. C. The clonal evolution of tumor cell populations. Science 194, 23-28 (1974).

18. Moxon, E. R., Rainey, P. B., Nowak, M. A. \& Lenski, R. E. Adaptive evolution of highly mutable loci in pathogenic bacteria. Curr. Biol. 4, 24-33 (1994).

19. Lederberg, S. Genetics of host-controlled restriction and modification of deoxyribonucleic acid in Escherichia coli. J. Bacteriol. 91, 1029-1036 (1966).

20. Carlton, B. C. \& Brown, B. J. in Manual of Methods for General Bacteriology (ed. Gerhardt, P.) 222-242 (American Society for Microbiology, Washington DC, 1981).

21. Luria, S. E. \& Delbrück, M. Mutations of bacteria from virus sensitivity to virus resistance. Genetics 28, 491-511 (1943)

22. Miller, J. H. A Short Course in Bacterial Genetics (Cold Spring Harbor Laboratory Press, NY, 1992).

23. Pang, P. P., Lundberg, A. S. \& Walker, G. C. Identification and characterization of the $m u t L$ and $m u t S$ gene products of Salmonella typhimurium LT2. J. Bacteriol. 163, 1007-1015 (1985).

24. Taucher-Scholz, G. \& Hoffman-Berling, H. Identification of the gene for DNA helicase II of Escherichia coli. Eur. J. Biochem. 137, 573-580 (1983).

25. Bhatnagar, S. K. \& Bessman, M. J. Studies on the mutator gene, mutT, of Escherichia coli. Molecula cloning of the gene, purification of the gene product, and identification of a novel nucleoside triphosphatase. J. Biol. Chem. 263, 8953-8957 (1988).

26. Horiuchi, T., Maki, H., Maruyama, M. \& Sekiguchi, M. Identification of the dnaQ gene product and location of the structural gene for RNAse $\mathrm{H}$ of Escherichia coli by cloning of the genes. Proc. Natl Acad. Sci. USA 78, 3770-3774 (1981).

27. Schaaper, R. M. \& Cornacchio, R. An Escherichia coli dnaE mutation with suppressor activity toward mutator mutD5. J. Bacteriol. 174, 1974-1982 (1992).

28. Sambrook, E. F., Fritsch, T. \& Maniatis, J. Molecular Cloning: A Laboratory Manual 2nd edn (Cold Spring Harbor Laboratory Press, NY, 1989).

29. Ma, W. T., Sandri, G. v. H. \& Sarkar, S. Analysis of the Luria-Delbrück distribution using discrete convolution powers. J. Appl. Prob. 29, 255-267 (1992).

30. Stewart, F. M. Fluctuation tests: how reliable are the estimates of mutation rates? Genetics 137, 1139 1146 (1994).

Acknowledgements. We thank F. Taddei for sharing data before publicaiton; C. Zeyl for permission to cite unpublished data; A. White for help with statistical analyses; B. Bohannan, L. Ekunwe and P. Frank for technical assistance; T. Cebula, S. F. Elena, D. G. MacPhee, J. Mongold and M. Travisano for discussions; and H. Maki and J. E. LeClerc for plasmids. Supported by the US NSF and by the Center for Microbia Ecology, Michigan State University.

Correspondence and requests for materials should be addressed to P.D.S. (e-mail: paulsnie@sas.upenn. edu).

\section{Evidence from Turner's syndrome of an imprinted X-linked locus affecting cognitive function}

\section{H. Skuse ${ }^{\star}$, R. S. James $\dagger$, D. V. M. Bishop $\ddagger$, B. Coppin $₫$, P. Dalton $\dagger$, G. Aamodt-Leeper ${ }^{\star}$, M. Bacarese-Hamilton ${ }^{\star}$, C. Creswell ${ }^{\star}$, R. McGurk ${ }^{\star} \&$ P. A. Jacobs $\dagger$}

* Behavioural Sciences Unit, Institute of Child Health, 30 Guilford Street, London WC1N 1EH, UK

$\dagger$ Wessex Regional Genetics Laboratory, Salisbury District Hospital, Salisbury, Wiltshire SP2 8BJ, UK

$\ddagger$ MRC Applied Psychology Unit, 15 Chaucer Road, Cambridge CB2 2EF, UK $\$$ Wessex Regional Genetics Service, Princess Anne Hospital, Coxford Road, Southampton SO16 5YA, UK

Turner's syndrome is a sporadic disorder of human females in which all or part of one $\mathrm{X}$ chromosome is deleted ${ }^{1}$. Intelligence is usually normal ${ }^{2}$ but social adjustment problems are common ${ }^{3}$. Here we report a study of 80 females with Turner's syndrome and a single $\mathrm{X}$ chromosome, in $\mathbf{5 5}$ of which the $\mathrm{X}$ was maternally derived $\left(45, X^{m}\right)$ and in 25 it was of paternal origin $\left(45, X^{p}\right)$. Members of the $45, X^{p}$ group were significantly better adjusted, with superior verbal and higher-order executive function skills, which mediate social interactions ${ }^{4}$. Our observations suggest that there is a genetic locus for social cognition, which is imprinted ${ }^{5}$ and is not expressed from the maternally derived X chromosome. Neuropsychological and molecular investigations of eight females with partial deletions of the short arm of the $\mathrm{X}$ chromosome $\mathrm{e}^{6}$ indicate that the putative imprinted locus escapes $\mathrm{X}$-inactivation ${ }^{7}$, and probably lies on $\mathrm{Xq}$ or close to the centromere on $\mathrm{Xp}$. If expressed only from the $\mathrm{X}$ chromosome of paternal origin, the existence of this locus could explain why 46,XY males (whose single $\mathrm{X}$ chromosome is maternal) are more vulnerable to developmental disorders of language and social cognition, such as autism, than are $46, \mathrm{XX}$ females ${ }^{8}$.

An increasing number of mammalian genes are known to be subject to genomic imprinting, defined as parental origin-specific differential gene expression ${ }^{5}$. No imprinted gene has yet been described on the $\mathrm{X}$ chromosome in humans ${ }^{9}$, although the Xist gene has been shown to be imprinted in the mouse ${ }^{10}$. We considered that it should be possible to identify the effects of an X-linked imprinted locus by comparing classes of females with Turner's syndrome. In this chromosomal disorder all, or a substantial part, of one $\mathrm{X}$ chromosome is missing as a result of non-disjunction (chromosome loss during gametogenesis or early cleavage of the zygote). In $70 \%$ of monosomic $(45, \mathrm{X})$ Turner-syndrome females, the single $\mathrm{X}$ chromosome is maternal in origin ${ }^{1}$; in the remainder it is paternal. Normal females $(46, \mathrm{XX})$ possess both a maternally derived $\mathrm{X}$ chromosome $\left(\mathrm{X}^{\mathrm{m}}\right)$ and a paternally derived $\mathrm{X}$ chromosome $\left(\mathrm{X}^{\mathrm{p}}\right)$, one of which is randomly inactivated in any given somatic cell ${ }^{7}$. In monosomy $\mathrm{X}$, the single chromosome is never inactivated. Differences in physical or behavioural phenotype between $45, \mathrm{X}^{\mathrm{p}}$ and $45, \mathrm{X}^{\mathrm{m}}$ Turner-syndrome subjects might therefore indicate the existence of an imprinted genetic locus.

Impaired social competence and adjustment are frequent in Turner's syndrome ${ }^{3}$, but a minority have good social skills ${ }^{11}$. Intelligence is usually normal in monosomic $(45, \mathrm{X})$ cases $^{2}$. We wished to test the hypothesis that $45, \mathrm{X}^{\mathrm{p}}$ females would be distinguishable from $45, \mathrm{X}^{\mathrm{m}}$ females by their social behaviour.

We karyotyped 80 monosomic $(45, \mathrm{X})$ females and eight with deletions of the short arm of one X chromosome (46,XXp-). The 